\title{
The Lay Public's Understanding and Perception of Dementia in a Developed Asian Nation
}

\author{
Wai Jia Tan ${ }^{a}$ Song-lee Hong ${ }^{b}$ Nan Luo ${ }^{c}$ Tong Jen Lo $^{d}$ \\ Philip Yape \\ ${ }^{a}$ Ministry of Health Holdings, ${ }^{b}$ Department of Social Work, Faculty of Arts and Social \\ Sciences, and 'Saw Swee Hock School of Public Health, National University of Singapore, \\ dPrimary and Community Care Division, Ministry of Health, and e Department of Geriatric \\ Medicine, Khoo Teck Puat Hospital, Singapore
}

\section{Key Words}

Dementia $\cdot$ Public awareness $\cdot$ Knowledge $\cdot$ Attitude $\cdot$ Perception $\cdot$ Stigma $\cdot$ Screening

\begin{abstract}
Background: Early detection of dementia aims to improve treatment outcomes. However, poor perception and understanding of dementia are significant barriers. We aim to investigate the public's perception of dementia and identify variables associated with the different profiles of public perception. Methods: A custom-designed questionnaire was used to assess laypersons' knowledge and perception of dementia during a health fair at a public hospital in Singapore, a developed Asian nation. Out of a sample of 370 subjects, 32 declined to participate (response rate $=91.4 \%$ ). Latent class analysis (LCA) was used to identify meaningful subgroups of subjects from significant associations with multiple indicators of dementia awareness. Multinomial logistic regression was performed exploring variables associated with each of the subgroups derived from LCA. Results: The majority of the study participants were female (66.9\%), 65 years or older (71.1\%), and ethnic Chinese (88.1\%). LCA classified the study participants into 3 subgroups: Class 1 (good knowledge, good attitude), Class 2 (good knowledge, poor attitude), and Class 3 (poor knowledge, poor attitude), in proportions of $14.28,63.83$, and $21.88 \%$, respectively. Compared to other classes, participants with good knowledge and good attitude towards dementia (Class 1 ) were more likely to know someone with dementia and understand the effects of the disease, be married, live in private housing, receive higher monthly income, and not profess
\end{abstract}


belief in Buddhism, Taoism, or Hinduism. Conclusion: Our results show that the public in Singapore may not be ready for screening initiatives and early dementia diagnosis. Education efforts should be targeted at lower socioeconomic groups, singles, and those of certain oriental religions.

Copyright $\odot 2012$ S. Karger AG, Basel

\section{Introduction}

With the ageing population, the prevalence of dementia is set to increase [1]. This has serious impacts on medical, social, and economic fronts [2]. The number of people with dementia is expected to double every 20 years and reach 81.1 million by 2040 [3]. It is projected that in the 21st century, the majority of the world's older people will be living in Asia $[4,5]$.

Persons with dementia present to health-care professionals when the disease is symptomatic. By the time symptomatic functional decline is detected, brain pathology is often advanced. This could explain the failure of clinical trials and hence the move towards a preclinical diagnosis of dementia [6-8]. It is hoped that efforts to increase awareness, reduce stigma, and clarify misunderstandings of the illness can enable early detection of dementia. Early diagnosis can help patients to plan for the future when insight is still retained and thus to better cope with the illness [9]. Early intervention has the potential to be cost-effective and can improve the quality of life for persons with dementia [10].

For early dementia detection initiatives to make a positive impact on society, they must be socially acceptable. Several studies have shown that high refusal rates for screening or further diagnostic tests for dementia may be related to poor attitudes, poor knowledge, and perceived stigma towards dementia $[9,11-13]$. Although the study participants perceived that early diagnosis may provide more time for planning for an individual's health, financial, and housing needs, they had concerns about losing health insurance coverage, driving privileges, and employment $[12,14]$. Moreover, there is still no cure for dementia.

Interethnic differences exist in dementia epidemiology [5], and differences in culture, beliefs, and religion colour the way dementia is perceived [15]. Previous studies on Chinese people, for example, showed that because of their inherent cultural beliefs, dementia symptoms may be normalized, and there is a tendency to hide their familial problems from the outside world [16]. In a predominantly Asian community such as Singapore, with the majority being Chinese, understanding the nuances behind the various sociocultural beliefs can help us to tailor strategies for public education and caregiver interventions, hence reducing the burden associated with dementia.

With the rapid progress in dementia diagnostics, presymptomatic detection of Alzheimer's disease may soon become a reality [17-19]. Nonetheless, in spite of the developments on the medical front, the public appears to be lukewarm to screening [20]. Without wide public acceptance for dementia screening, pushing the frontiers of early diagnosis may be premature. Therefore, we conducted a survey with members from the public to assess their knowledge and attitudes towards dementia, including their perceived stigma of the illness. The results of the study will contribute to our understanding of how dementia is perceived in an Asian society and help focus our public education efforts to bridge the chasm between rapid medical progress and the seemingly more conservative attitudes of the public towards dementia. 


\section{Methods}

\section{Subjects and Procedure}

Ethics approval for this study was obtained from the Domain Specific Review Board of the National Healthcare Group (NHG) Singapore. The information used for the study was collected during a health fair open to the general public conducted in conjunction with the opening of a new hospital, Khoo Puat Hospital, in the north of Singapore. A booth was set up to provide information on dementia, and a custom-designed questionnaire was used to elicit responses on the knowledge and attitudes of the layperson towards the disease. The responses to the questionnaire were used as a basis for providing customized education. The protocol administrator explained the nature and aims of the questionnaire, the voluntary nature of participation, and the confidentiality of the responses to consecutive members of the public who came by the booth. Verbal consent was obtained for the responses to be used for research, but no written consent was taken as anonymity was maintained for the information collected.

Inclusion criteria included (1) literacy in English or Mandarin and ability to complete a survey questionnaire, and (2) age greater than 18 years. Although the survey questionnaire was designed to be self-administered, the subjects were first given an explanation and rundown of the questionnaire before being left to answer on their own. All questionnaires were completed on site.

\section{Measurements}

Demographics

Age, gender, race, marital status, education, housing type, household income per month, and religion were obtained. To capture the participants' personal experience with a person with dementia, two questions were asked: (1) whether they knew of anyone (i.e. relatives, friends, or someone from the workplace) diagnosed with dementia, and (2) whether they understood the effects and consequences of dementia as a result of knowing the person with dementia (table 1).

Awareness of Dementia: Dementia Knowledge and Attitude

To measure the public awareness of dementia, we defined its two domains: knowledge of dementia and attitude towards dementia. Nine statements (five on knowledge and four on attitude) were presented with 'yes' and 'no' response options to assess the participants' knowledge of dementia and their attitude towards the disease. These are detailed in table 1. Out of the four statements assessing attitude, two pertained to attitude with respect to treatment, while the other two were concerned with having a stigma-free attitude towards dementia. The content of the questionnaire was finalised on the basis of a literature search for questionnaires assessing dementia knowledge and attitudes [14,21,22], as well as relevance to our local sociocultural context. At the same time, expert opinion from three professionals (nurse, social worker, and geriatrician) steeped in dementia care, with a mean of 8-year working experience in the field, was obtained (table 2).

\section{Statistical Analysis}

Latent class analysis (LCA) is a person-centred analysis for identifying subgroups of cases from multiple indicators of the issue of interest. For our study, LCA assigns the study participants to subgroups based on the associations between variables of dementia awareness (knowledge and attitudes). This statistical technique extracts a meaningful connotation from significant associations with multiple indicators of dementia awareness, which can overcome a complexity from simple cross-tabulations [23]. Compared to a traditional vari- 
Table 1. Measurement of dementia awareness $(n=329)$

\begin{tabular}{|c|c|c|c|}
\hline & & Participants, & n $(\%)$ \\
\hline & & correct & incorrect \\
\hline Dementia & 1. Is memory decline due to ageing the same as dementia? & $133(40.43)$ & $196(59.57)$ \\
\hline knowledge & 2. Is dementia part of normal ageing such that all older & & \\
\hline & people develop dementia as they age? & $171(51.98)$ & $158(48.02)$ \\
\hline & $\begin{array}{l}\text { 3. Based on what we know about dementia, the person } \\
\text { diagnosed with dementia should be concerned about it and }\end{array}$ & & \\
\hline & what is to come. & $271(82.37)$ & $58(17.63)$ \\
\hline & 4. People with mild dementia will eventually progress to & & \\
\hline & $\begin{array}{l}\text { moderate and severe dementia with time. } \\
\text { 5. A person with advanced dementia may no longer be able }\end{array}$ & $234(71.12)$ & $95(28.88)$ \\
\hline & to talk, walk, recognise himself or his loved ones. & $220(66.87)$ & $109(33.13)$ \\
\hline Attitude & 1. If you have dementia, you would rather not know. & $65(19.76)^{\mathrm{a}}$ & $264(80.24)^{\mathrm{b}}$ \\
\hline & $\begin{array}{l}\text { 2. Would you make plans for the future (for yourself and } \\
\text { family) if diagnosed with dementia? }\end{array}$ & $108(32.83)^{\mathrm{a}}$ & $221(67.17)^{\mathrm{b}}$ \\
\hline & 3. If you have dementia, you would not want others to know. & $205(62.31)^{c}$ & $124(37.69)^{\mathrm{d}}$ \\
\hline & embarrassed. & $249(75.68)^{\mathrm{c}}$ & $80(24.32)^{\mathrm{d}}$ \\
\hline
\end{tabular}

${ }^{a}$ Positive; ${ }^{b}$ negative; ${ }^{\mathrm{c}}$ stigma-free; ${ }^{\mathrm{d}}$ stigmatized.

able-centred analysis, the LCA can draw a parsimonious person-centred conceptualization of the dementia awareness patterns.

In this study, Mplus 3.0 was used to classify homogenous subgroups of the study participants by estimating similarities in the nine items of dementia awareness. Using the LCA with dichotomous responses, two sets of parameters were estimated [24]: (a) latent class probabilities indicate the number of classes and relative size of each class, and (b) the individual class members' means and variances of observable indicators are estimated in each of the latent classes.

The optimal number of class enumeration is resolute by comparing estimated solutions of increasing numbers of classes. Modelling processes continue until the optimal number of classes is successfully fixed by multiple statistical fit indices. To corroborate an optimal class solution, each of the models was executed by stepwise addition of classes until the model fit the data, with a significant visual representation of patterns and a goodness of fits including the smallest scores on the Akaike Information Criterion (AIC) and Bayesian Information Criterion (BIC) [25], as well as a higher score in entropy. The Vuong-Lo-Mendell-Rubin test [26] was used to examine the optimal number of classification. As a single latent variable with a set of underlying 'classes', the dementia awareness pattern was entered into the final analytic model.

As a nominal outcome variable, the pattern of dementia awareness estimated by the LCA was entered into a multinomial logistic regression. Stata 10 was used to examine determinants associated with the pattern of dementia awareness. Before entering independent variables in the multinomial logistic regression, bivariate analyses ( $\chi^{2}$ tests) identified significant associations between independent variables and the three classes of dementia awareness (table 2).

Except for the variable 'income', all study variables had missing values of less than $5 \%$ of observations. No statistically significant differences were found between the study participants who missed or did not miss any responses. To minimize bias from the uncertainty of 'missingness', a hot-decking imputation procedure [27] was applied to complete the missing values. 


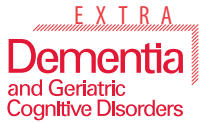

\begin{tabular}{l}
\hline Dement Geriatr Cogn Disord Extra 2012;2:433-444 \\
\begin{tabular}{l|l}
\hline DOI: $10.1159 / 000343079$ & $\begin{array}{l}\text { C } 2012 \text { S. Karger AG, Basel } \\
\text { www.karger.com/dee }\end{array}$ \\
Published online: October 18, 2012 &
\end{tabular}
\end{tabular}

Tan et al.: The Lay Public's Understanding and Perception of Dementia in a Developed

Asian Nation

Table 2. Descriptive statistics of the study variables by dementia awareness patterns $(\mathrm{n}=329)$

\begin{tabular}{|c|c|c|c|c|c|}
\hline \multirow[t]{2}{*}{ Variables } & \multicolumn{3}{|c|}{ Participants, n (\%) } & \multirow{2}{*}{$\begin{array}{l}\text { Statistics } \\
\text { Pearson's } \\
\chi^{2} \text { (d.f.) }\end{array}$} & \multirow{2}{*}{$\begin{array}{l}\text { Total } \\
\text { sample } \\
\mathrm{n}(\%)\end{array}$} \\
\hline & Class 1 & Class 2 & Class 3 & & \\
\hline \multicolumn{6}{|l|}{ Personal experience with a person with dementia } \\
\hline Do not know anyone with dementia & $29(12.13)$ & $148(61.92)$ & $62(25.94)$ & \multirow{3}{*}{$\begin{array}{l}\chi^{2}(4)=14.42 \\
p=0.006\end{array}$} & $239(72.64)$ \\
\hline Know someone with dementia but do not & & & & & \\
\hline know the effects of dementia & $3(13.04)$ & $14(60.87)$ & $6(26.09)$ & & $23(6.99)$ \\
\hline Know someone with dementia and & & & & & \\
\hline know the effects of dementia & $15(22.39)$ & $48(71.64)$ & $4(5.97)$ & & $67(20.36)$ \\
\hline \multicolumn{6}{|l|}{ 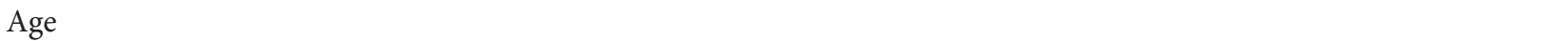 } \\
\hline$>65$ years & $32(13.68)$ & $150(64.10)$ & $52(22.22)$ & \multirow{2}{*}{$\begin{array}{l}\chi^{2}(2)=0.26 \\
p=0.876\end{array}$} & $234(71.12)$ \\
\hline$\leq 65$ years & $15(15.79)$ & $60(63.16)$ & $20(21.05)$ & & $95(28.88)$ \\
\hline \multicolumn{6}{|l|}{ Gender } \\
\hline Female & $29(13.18)$ & $144(65.45)$ & $47(21.36)$ & \multirow{2}{*}{$\begin{array}{l}\chi^{2}(2)=0.92 \\
p=0.633\end{array}$} & $220(66.87)$ \\
\hline Male & $18(16.51)$ & $66(60.55)$ & $25(22.94)$ & & $109(33.13)$ \\
\hline \multicolumn{6}{|l|}{ Race } \\
\hline Chinese & $42(14.48)$ & $187(64.48)$ & $61(21.03)$ & \multirow{2}{*}{$\begin{array}{l}\chi^{2}(2)=1.04 \\
p=0.595\end{array}$} & $290(88.15)$ \\
\hline Non-Chinese & $5(12.82)$ & $23(58.97)$ & $11(28.21)$ & & $39(11.85)$ \\
\hline \multicolumn{6}{|l|}{ Marital status } \\
\hline Married & $38(15.08)$ & $159(63.10)$ & $55(21.83)$ & \multirow{2}{*}{$\begin{array}{l}\chi^{2}(2)=0.57 \\
p=0.753\end{array}$} & $252(76.60)$ \\
\hline Unmarried & $9(11.69)$ & $51(66.23)$ & $17(22.08)$ & & $77(23.40)$ \\
\hline \multicolumn{6}{|l|}{ Education } \\
\hline Primary/minimal & $2(9.52)$ & $17(80.95)$ & $2(9.52)$ & \multirow{3}{*}{$\begin{array}{l}\chi^{2}(4)=13.38 \\
p=0.009\end{array}$} & $21(6.38)$ \\
\hline 'O' and 'A' level & $24(11.48)$ & $132(63.16)$ & $52(25.36)$ & & $225(68.39)$ \\
\hline Tertiary/diploma & $20(24.10)$ & $52(62.65)$ & $11(13.25)$ & & $83(25.23)$ \\
\hline \multicolumn{6}{|l|}{ House type } \\
\hline Private & $15(41.67)$ & $17(47.22)$ & $4(11.11)$ & \multirow{2}{*}{$\begin{array}{l}\chi^{2}(2)=25.10 \\
\mathrm{p}<0.001\end{array}$} & $36(10.94)$ \\
\hline Public & $32(10.92)$ & $193(65.87)$ & $68(23.21)$ & & $293(89.06)$ \\
\hline \multicolumn{6}{|l|}{ Monthly household income group } \\
\hline$<$ USD 2,500 & $30(13.64)$ & $133(60.45)$ & $57(25.91)$ & \multirow{2}{*}{$\begin{array}{l}\chi^{2}(2)=6.29 \\
p=0.04\end{array}$} & $220(66.87)$ \\
\hline$\geq$ USD 2,500 & $17(15.60)$ & $77(70.64)$ & $15(13.76)$ & & $109(33.13)$ \\
\hline \multicolumn{6}{|l|}{ Religion } \\
\hline Buddhist/Hindu/Taoist & $7(4.79)$ & $99(67.11)$ & $40(27.40)$ & \multirow{3}{*}{$\begin{array}{l}\chi^{2}(4)=37.85 \\
p<0.001\end{array}$} & $146(44.38)$ \\
\hline Christian/Catholic & $28(34.57)$ & $42(51.85)$ & $11(13.58)$ & & $111(33.74)$ \\
\hline Free-thinker & $10(13.89)$ & $48(66.67)$ & $14(19.44)$ & & $72(21.88)$ \\
\hline
\end{tabular}

\section{Results}

\section{Sample Characteristics}

A total of 370 subjects satisfied the inclusion criteria and were invited to participate. Thirty-two refused, leaving 338 (91.4\%) who provided verbal consent and completed the questionnaire. After excluding subjects with significant missing data, the final analysable sample was 329 . We could not compare the characteristics of the respondents with the nonrespondents as no information on the latter was captured. The majority of the study participants were female (66.9\%), 65 years or older (71.1\%), and ethnic Chinese (88.1\%). In response to the questions regarding experience with dementia, $27.4 \%$ of the sample endorsed that they knew someone with dementia. Detailed characteristics of the study sample are displayed in table 2 . 
Tan et al.: The Lay Public's Understanding and Perception of Dementia in a Developed

Asian Nation

Table 3. Statistical model fits of two- to four-class solutions of dementia awareness patterns

\begin{tabular}{lrrr}
\hline Model fits & 2 classes & 3 classes & \multicolumn{1}{c}{4 classes } \\
\hline AIC & $7,758.534$ & $7,634.677$ & $7,599.731$ \\
BIC & $7,870.241$ & $7,802.238$ & $7,823.147$ \\
Entropy & 0.954 & 0.945 & 0.775 \\
Vuong-Lo-Mendell-Rubin likelihood ratio test & 224.805 & 181.496 & 59.727 \\
\hline p value & $\mathrm{p}<0.001$ & $\mathrm{p}<0.001$ & $\mathrm{p}=0.3659$ \\
\hline
\end{tabular}

\section{Dementia Knowledge and Attitude}

Table 1 shows a breakdown of the responses to the questionnaire. Close to $60 \%$ of the study participants incorrectly answered that memory decline is the same as dementia, and $48 \%$ considered dementia to be a normal ageing process. The attitude towards dementia was generally negative, with $80.2 \%$ choosing not to know the diagnosis of dementia and $67.2 \%$ avoiding advanced care planning if diagnosed. With regards to stigma, close to one quarter $(24.3 \%)$ and one third (37.7\%) of the study participants would feel ashamed of having dementia or want to hide it from others, respectively.

\section{Pattern of Dementia Awareness}

All classifications among the two- through four-class enumerations showed an inconsistent incidence across indicators of dementia awareness (table 3). Each of the models was assessed through illustrated delineations of class profiles as well as statistical model fits for interpretability. Two- and three-class models showed more apparent theoretical distinctions among indicators. Of the three classifications, the three-class solution was the optimal fit, indicating the lower score in AIC $(7,634.677)$ and lowest BIC $(7,802.238)$ as well as higher score in entropy (0.945). A Vuong-Lo-Mendell-Rubin likelihood ratio test demonstrated that the three-class solution improved the two-class model, whereas the four-class solution did not advance the three-class model (table 3). Figure 1 illustrates the pattern of dementia awareness with the three-class solution on nine itemized dementia knowledge, positive attitude towards dementia preparedness, and stigma of dementia.

The study participants were classified into three groups: Class 1 (good knowledge, good attitude), Class 2 (good knowledge, poor attitude), and Class 3 (poor knowledge, poor attitude). Except for one indicator regarding stigma of dementia (Stigma-free 1 in fig. 1), all indicators in the hypothesized three domains significantly contributed to distinguishing the pattern of dementia awareness. Overall, its pattern was fairly hierarchical in the conditional probabilities of five indicators of dementia knowledge and was separated into two obvious groups in the conditional probabilities of two indicators of positive attitude and two indicators of stigma-free attitude.

Class 1 ( $\mathrm{n}=47 ; 14.28 \%)$ was characterized as having consistently higher conditional probabilities across all nine indicators of dementia awareness, as compared to the other two classes. Those who were classified in this group reported better knowledge of dementia, a more positive attitude and less stigma. Compared to Class 1 subjects, Class 2 subjects $(\mathrm{n}=$ $210 ; 63.83 \%$ ) showed fair knowledge levels but a more negative attitude to dementia and relatively higher stigma. Interestingly, members of this class were more likely to recognize that people with mild dementia will progress to severe dementia with time, and were least likely to show positive attitudes to dementia. Class 3 subjects $(n=72 ; 21.88 \%)$ reported the lowest conditional probabilities in all domains of knowledge, and a positive and stigma-free atti- 
Fig. 1. The pattern of dementia awareness. Higher conditional probabilities on the $y$-axis mean better knowledge, more positive attitude, and less stigma against dementia.

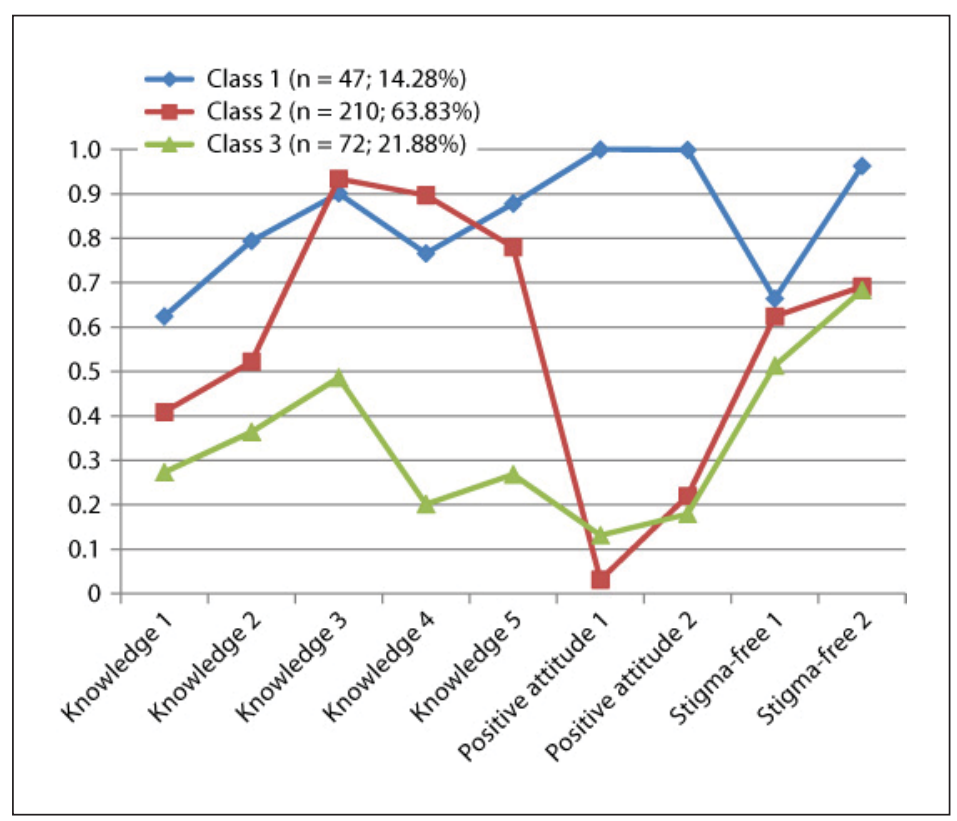

tude. Thus, those in Class 3 were more likely to have poor knowledge, negative attitudes, and higher stigma.

Factors Associated with the Pattern of Dementia Awareness

Using a multinomial logistic regression on the pattern of dementia awareness classified with three categories estimated by the LCA, we estimated its associated factors (table 4). Compared with those who did not know anyone with dementia, those who knew someone with dementia and knew the effects of dementia were more likely to be in Class 1 than Class 3. They were also more likely to be classified in Class 2 than Class 3.

The sociodemographics of the study participants were significant contributors to distinguishing the patterns of dementia awareness. Specifically, those who are married were more likely to be in Class 1 than Class 3. Those who are living in public housing were less likely to be in Class 1 than Class 2 or Class 3. Those who received more than USD 2,500 household income per month were more likely to be in Class 1 or Class 2 than Class 3. Compared to Buddhists, Hindus, and Taoists, Christians and free-thinkers were more likely to be classified in Class 1 than Class 2 or Class 3 (table 4).

\section{Discussion}

In spite of efforts to raise awareness about dementia in recent years, the results suggest that a substantial proportion of our population still do not have an accurate understanding of dementia. Most people see dementia as part of the normal ageing process. As many as $59.6 \%$ of the study participants answered incorrectly when asked if memory decline due to ageing is the same as dementia, and almost half (48.0\%) of them gave incorrect responses when asked whether dementia is part of normal ageing.

This 'normalization' of dementia symptoms could largely be influenced by culture. Culture affects how meaning is assigned to the illness [28]. In a study on a multiethnic sample of Asian-American caregivers in the United States [29], it was found that different models 
Table 4. Multinomial logistic regression of caregivers' coping styles $(\mathrm{n}=329)$

\begin{tabular}{|c|c|c|c|c|c|c|}
\hline \multirow[t]{2}{*}{ Variables } & \multicolumn{2}{|l|}{ Class $1^{\mathrm{a}}$} & \multicolumn{2}{|l|}{ Class $1^{\mathrm{b}}$} & \multicolumn{2}{|c|}{ Class $3^{\mathrm{b}}$} \\
\hline & OR & $\mathrm{CI}$ & OR & $\mathrm{CI}$ & OR & $\mathrm{CI}$ \\
\hline \multicolumn{7}{|l|}{ Personal experience with a person with dementia } \\
\hline \multicolumn{7}{|l|}{ Know someone with dementia but do not } \\
\hline know the effects of dementia & 0.92 & $0.17-4.81$ & 0.92 & $0.21-4.04$ & 1.00 & $0.36-2.81$ \\
\hline \multicolumn{7}{|l|}{ Know someone with dementia and know the } \\
\hline effects of dementia & $8.96^{* *}$ & $2.48-32.35$ & 1.80 & $0.79-4.11$ & $0.20^{* *}$ & $0.07-0.59$ \\
\hline Age $(>65$ years $)$ & 1.58 & $0.60-4.17$ & 1.12 & $0.48-2.59$ & 0.71 & $0.37-1.36$ \\
\hline Male & 0.75 & $0.29-1.87$ & 1.09 & $0.49-2.39$ & 1.46 & $0.78-2.72$ \\
\hline Chinese & 0.77 & $0.20-2.94$ & 0.55 & $0.17-1.79$ & & \\
\hline Married & $3.04^{*}$ & $0.99-9.32$ & 2.09 & & 0.69 & $0.33-1.44$ \\
\hline \multicolumn{7}{|l|}{ Education } \\
\hline 'O' and 'A' level' & 1.06 & $0.22-5.08$ & 1.54 & $0.39-6.06$ & 1.45 & $0.55-3.86$ \\
\hline Tertiary/diploma ${ }^{c}$ & 2.24 & $0.41-12.26$ & 2.18 & $0.51-9.27$ & 0.97 & $0.32-2.98$ \\
\hline House type (public housing) & $0.29 *$ & $0.08-1.01$ & $0.34^{*}$ & $0.13-0.86$ & 1.13 & $0.36-3.61$ \\
\hline Monthly household income group ( $\geq$ USD 2,500 ) & 1.22 & $0.43-3.44$ & 0.60 & $0.25-1.42$ & $0.50^{*}$ & $0.24-1.02$ \\
\hline \multicolumn{7}{|l|}{ Religion } \\
\hline Christian/catholic & $9.57^{* * *}$ & $3.21-28.57$ & $7.68^{* * *}$ & $3.12-18.96$ & 0.80 & $0.35-1.81$ \\
\hline Free-thinker & $2.85^{*}$ & $0.97-9.17$ & $2.59^{*}$ & $0.98-7.22$ & 0.91 & $0.43-1.89$ \\
\hline Model fit & \multicolumn{6}{|c|}{ Logistic regression $\chi^{2}(24)=75.71^{* * *}$; pseudo $\mathrm{R}^{2}=0.128$} \\
\hline
\end{tabular}

${ }^{a}$ The reference group is Class 3. ${ }^{b}$ The reference group is Class $2 .^{c}$ The reference group is a primary education group. $\mathrm{d}$ The reference group is a Buddhist/Hindu/Taoist group. ${ }^{*} \mathrm{p}<0.05 ;{ }^{* *} \mathrm{p}<0.01 ;{ }^{* *} \mathrm{p}<0.001$.

exist in the conceptualization of dementia. While the biomedical model perceived dementia more as a medical disease, the folk model viewed its symptoms as part of the normal consequence of growing old. Labels such as 'confused', 'memory deterioration', or 'having a difficult personality' were used to describe dementia symptoms. In the mixed medical-folk model, dementia was referred to with biomedical labels such as Alzheimer's disease, but folk models of illness were used to explain it.

In Asian countries, the influence of traditional Chinese folk culture and religions such as Buddhism and Taoism [30] can colour the way people view illness, leading them to see illness as an inevitable part of life, hence the lack of necessity to pursue a diagnosis and seek treatment $[15,31]$. They may also believe that the cause of illness is related to their destiny and sealed in their fate. As such, it is not surprising that in an Asian population with a strong Chinese heritage, a large proportion of people may normalize dementia symptoms. Culturally congruent educational interventions to reach out to different sociodemographic and ethnic communities are therefore necessary.

Despite the knowledge gaps, the majority understood the severe consequences of dementia. In total, $71 \%$ of the study participants knew that people with mild dementia would eventually progress to moderate and severe dementia with time; $66.9 \%$ knew that in advanced dementia, the person may no longer talk, walk, or recognize their loved ones. However, this was not accompanied by a proactive response to the illness, and it was a largely negative attitude that prevailed. The majority $(80.2 \%)$ said they would rather not know about their diagnosis of dementia, and only $32.8 \%$ would make plans for the future if they or one of their family members were diagnosed with the illness. In the same vein, studies in the US have found that, while study participants agreed to be screened for dementia, a large proportion of those who screened positive did not return for further follow-up [7]. It has been argued 
that dementia screening can allay fears, especially if the test result is negative. Negative attitudes in the public could indeed be due to fears associated with the diagnosis of dementia. A sense of fatalism, beliefs about being diagnosed, and awareness of poor self-efficacy could all account for these fears [32]. Without doubt, the benefits of early treatment would need to be emphasized in any public education effort in dementia.

Several other reasons could account for the negativity. They can be explained by an individual's coping styles and the prevailing attitudes towards old age and 'senility'. Avoidance coping, denial and a fear of what may come, ageism and 'dementiaism', are possible reasons behind the findings and certainly warrant more in-depth study. Stigma associated with dementia was also investigated, and significant proportions of 37.7 and $24.3 \%$ of the study participants claimed that they would not want others to know of their diagnosis and would be ashamed or embarrassed, respectively. While it is heartening to note that a greater proportion of the participants are 'stigma-free', about one third still expressed shame with the disease, calling for more efforts at removing stigma associated with dementia.

A substantial portion of the participants $(63.83 \%)$ who had adequate knowledge about dementia were found to have poor attitudes towards the disease (Class 2). Those who knew someone with dementia and its effects were more likely to be in Class 1 or Class 2 than Class 3 , implying that this group certainly had better knowledge. However, they were not more likely to be in Class 1 than Class 2, again indicating that adequate knowledge did not amount to having a positive attitude about dementia. We surmise that knowing about the disease may have divergent effects. While it is hoped that learning more about the illness and its serious effects can lead to better preparedness and preemptive coping plans, it could also instil fear, anxiety, and an avoidance of the issue.

The way people interpret events is crucial for whether they can cope with a situation successfully or become stressed by it [33]. This would imply that public education must go further than merely conveying information to the masses. An in-depth approach is needed whereby concerted efforts need to be undertaken to nurture good attitudes and perceptions towards dementia, and empower people to improve their self-efficacy. Reassurance with a supportive health-care infrastructure may also be helpful. Focus groups discussions and support groups may be a more effective means to address underlying negativities towards the illness.

The 2006 survey conducted by the MetLife Foundation [34] reported that, overall, 63\% of their study participants were concerned about having to provide care for someone with Alzheimer's disease [35]. Our study shows that compared with singles, married couples are more likely to have better knowledge, attitudes, and less stigma towards dementia. The married subjects could be more conscious of the prospect of themselves or their spouse succumbing to dementia in the future, given their sense of responsibility and commitment towards their family. Married people are also more likely to have more elderly dependents at risk of dementia and could be more aware of their filial duty towards them in their old age, especially in an oriental society like ours.

A Singapore study found that religion and spirituality have a positive effect on coping for family caregivers of persons with dementia [36]. Holding the belief that a higher being is in control of what happens in one's life can promote resilience and reduce depressive symptoms $[37,38]$. Religion and spirituality can affect knowledge and perceptions in different ways. Christians and free-thinkers, seemingly disparate in make-up, were more likely to be classified in Class 1, indicative of good knowledge and attitude, compared to the rest. We surmise that the commonality in the other oriental religions, namely, Buddhism, Hinduism, and Taoism, is the concept of 'karma' and reincarnation, where one's deeds in the past determine the present outcome. Christians and free-thinkers, however, do not subscribe to this belief. The cultural or spiritual belief that one could be partially responsible for 'bringing it upon oneself' could also explain the less positive attitude and greater stigma seen among 
Buddhists, Hindus, and Taoists. Finally, in practitioners of these religions, there is a tendency to resign their future to 'destiny'. A reluctance to actively alter one's destiny could also explain a predilection to shy away from wanting to know the diagnosis of dementia or make plans for the future.

In a study investigating the emotional reactions of Arab laypersons to a person with Alzheimer's disease, feelings of rejection were mainly associated with lower education and lower income [39]. This is similar to our findings, where study participants who received less than SGD 3,000 household income per month were more likely to be in Class 3, and those who were living in public housing were less likely to be in Class 1 . It is not surprising that those with a higher socioeconomic status have better knowledge and attitudes towards dementia. This group is likely to be more educated and informed on the popular issues of the times compared to the less well-off people who have to first contend with life's basic necessities. This phenomenon is commonly seen in other spectres of life as well [40]. Previous research has shown that due to communication barriers, those from lower income groups rely heavily on interpersonal sources to share and receive health information [41]. Thus, efforts can be put into tailoring word-of-mouth health communication styles among those in the lower-income groups to reduce communication inequities. This may help to reduce health disparities in the population.

Our findings clearly show that much needs to be done to improve the lay public's understanding of dementia. Given the rapid progress in early diagnosis of Alzheimer's disease, the lay community does not appear ready for screening initiatives and diagnosis of pre-symptomatic disease. LCA has enabled a better understanding of the profiles of the different classes of the study participants. Analysing these classes alongside their demographic characteristics allows some postulations to be made and enables a more focused approach to intervention to improve public awareness and attitudes towards dementia.

The findings imply greater efforts should be directed at singles, lower socioeconomic groups, and believers of Buddhism, Taoism, and Hinduism. Initiating dementia awareness programmes in schools may be a viable solution to start public education early and include as wide a population catchment as possible. Having dementia advocacy initiatives targeted at specific religious groups may also be helpful, and allowing persons with dementia and caregivers opportunities to share their experiences can help improve attitudes and reduce stigma.

Nonetheless, there are some limitations to our study, with the modest number of subjects and disproportionate representation from Chinese being one. We lack information from our minority ethnic groups such as the Indians and Malays. Also, as the surveys were conducted at a geriatric booth at a health fair in a public hospital, participants were more likely to have some interest in geriatric issues. Therefore, the negative attitudes towards dementia reflected in the study results could be an underestimate of the real situation.

The variable 'personal experience with a person with dementia' may include quite a heterogeneous group of people as the degree of interaction with a person of dementia could vary significantly, depending on whether he or she was a caregiver or a distant acquaintance with a person with the illness. With a larger number of participants, it would be interesting to break down the degree of personal experience and explore its effect on the outcomes. Finally, the division of monthly household income categories was guided by the median household income of Singaporeans, estimated to be USD 4,700 in 2010 [42]. We decided to adopt this value to define low income as half of the amount which would approximate USD 2,500, and this value was close to the median monthly household income of citizens staying in the smaller category of public housing (3-room flats). We acknowledge some arbitrariness was inherent in determining the low income cut-off, and thus the results may have varied had a different cut-off been chosen. 


\section{Acknowledgements}

We wish to express our gratitude to the staff and nurses of the geriatric clinic in Khoo Teck Puat Hospital for their kind assistance in administering the questionnaire to the participants. In particular, we would like to thank Jessie Tan, Chionh Hui Ling, and operations executive Woo Wan Ling.

\section{References}

1 Larson EB, Langa KM: The rising tide of dementia worldwide. Lancet 2008;372:430-432.

2 Cotter VT: The burden of dementia. Am J Manag Care 2007;13(suppl 8):S193-S197.

-3 Ferri CP, Prince M, Brayne C, et al: Global Prevalence of dementia: a Delphi consensus study. Lancet 2005;366:2112-2117.

4 Alzheimer's Disease International: World Alzheimer Report 2010: The Global Economic Impact of Dementia. London, Alzheimer's Disease International, 2010.

-5 Venketasubramanian N, Sahadevan S: Interethnic differences in dementia epidemiology: global and Asia-Pacific perspectives. Dement Geriatr Cogn Disord 2010;30:492-498.

-6 Jack CR Jr, Weigand SD, Shiung MM, et al: Atrophy rates accelerate in amnestic mild cognitive impairment. Neurology 2008;70:1740-1752.

7 Bonetta L: Screening Tests Are Accurate, But Patients Don't Follow Up. Alzheimer Research Forum. http://www.alzforum.org/new/detail.asp?id=2999 (last accessed March 20, 2012).

8 Petersen RC: Mild cognitive impairment. N Engl J Med 2011;364:2227-2234.

9 Werner P: Preparedness for Alzheimer's disease and its determinants among laypersons in Israel. Int Psychogeriatr 2012;24:205-211.

-10 Banerjee S, Wittenberg R: Clinical and cost effectiveness of services for early diagnosis and intervention in dementia. Int J Geriatr Psychiatry 2009;24:748-754.

-11 Hurt CS, Burns A: Why don't older adults with subjective memory complaints seek help? Int J Geriatr Psychiatry 2012;27:394-400.

-12 Boustani MA, Justiss MD, Frame A, Austrom MG, Perkins AJ, Cai X, Sachs GA, Torke AM, Monahan P, Hendrie HC: Caregiver and non-caregiver attitudes toward dementia screening. J Am Geriatr Soc 2011;59:681-686.

-13 Jones RW, Mackell J, Berthet K, Knox S: Assessing attitudes and behaviours surrounding Alzheimer's disease in Europe: key findings of the Important Perspectives on Alzheimer's Care and Treatment (IMPACT) survey. J Nutr Health Aging 2010;14:525-530.

- 14 Boustani M, Perkins AJ: measuring primary care patients' attitudes about dementia screening. Int J Geriatr Psychiatry 2008;23:812-820.

-15 Chan SW: Family caregiving in dementia: the Asian perspective of a global problem. Dement Geriatr Cogn Disord 2010;30:469-478.

-16 Prince M: Care arrangements for people with dementia in developing countries. Int J Geriatr Psychiatry 2004;19:170-177.

-17 Apostolova LG, Dutton RA, Dinov ID, et al: Conversion of mild cognitive impairment to Alzheimer disease predicted by hippocampal atrophy maps. Arch Neurol 2006;63:693-699; erratum 2007;64: $1360-1361$.

-18 Shaw LM, Vanderstichele H, Knapik-Czajka M, et al: Cerebrospinal fluid biomarker signature in Alzheimer's disease neuroimaging initiative subjects. Ann Neurol 2009;65:403-413.

-19 Forsberg A, Engler H, Almkvist O, et al: PET imaging of amyloid deposition in patients with mild cognitive impairment. Neurobiol Aging 2008;29:1456-1465.

20 Iliffe S, Wilcock J, Haworth D: Obstacles to shared care for patients with dementia: a qualitative study. Fam Practice 2006;23:353-362.

-21 Dieckmann L, Zarit SH, Zarit JM, Gatz M: The Alzheimer’s Disease Knowledge Test. Gerontologist 1988;28:402-407.

-22 Carpenter BD, Balsis S, Otilingam PG, Hanson PK, Gatz M: The Alzheimer's Disease Knowledge Scale: development and psychometric properties. Gerontologist 2009;49:236-247. 
23 McCutcheon AL: Basic concepts and procedures in single and multiple group latent class analysis; in Hagenaars JA, McCutcheon AL (eds): Applied Latent Class Analysis. Cambridge, Cambridge University Press, 2002, pp 56-87.

-24 Muthén BO: Beyond SEM: general latent variable modeling. Behaviormetrika 2002;29:81-117.

-25 Kass RE, Wasserman L: A reference Bayesian test for nested hypotheses and its relationship to the Schwartz criterion. J Am Stat Assoc 1995;90:928-934.

- 26 Lo Y, Mendell NR, Rubin DB: Testing the number of components in a normal mixture. Biometrika 2001;88:767-778.

27 Kotz S, Johnson NL, Read CB: Encyclopedia of Statistical Sciences. New York, Wiley, 2005.

- 28 Gilbert P, Bhundia R, Mitra R, McEwan K, Irons C, Sanghera J: Cultural differences in shame-focused attitudes towards mental health problems in Asian and non-Asian student women. Ment Health Relig Cult 2007;10:127-141.

-29 Hinton L, Franz CE, Yeo G, Levkoff SE: Conceptions of dementia in a multi-ethnic sample of family caregivers. J Am Geriatr Soc 2005;53:1405-1410.

-30 Mok E, Lai CKY, Wong FLF, Wan P: Living with early-stage dementia: the perspective of older Chinese people. J Adv Nurs 2007;59:591-600.

-31 Sethabouppha H, Kane C: Caring for the seriously mentally ill in Thailand: Buddhist family caregiving. Arch Psychiatr Nurs 2005;19:44-57.

- 32 Von Wagner C, Good A, Whitaker KL, Wardle J: Psychosocial determinants of socioeconomic inequalities in cancer screening participation: a conceptual framework. Epidemiol Rev 2011;33:135-147.

-33 Antonovsky A: The structure and properties of the sense of coherence scale. Soc Sci Med 1993; 36:725-733.

34 MetLife Foundation: Americans fear Alzheimer's more than heart disease, diabetes or stroke, but few prepare. http://www.prnewswire.com/news-releases/us-adults-fear-alzheimers-more-than-heartdisease-diabetes-or-stroke-but-few-prepare-56323872.html (last accessed March 20, 2012).

- 35 Anderson LA, Day KL, Beard RL, Reed PS, Wu B: The public's perceptions about cognitive health and Alzheimer's disease among the U.S. population: a national review. Gerontologist 2009;49(suppl 1):S3-S11.

-36 Lim J, Griva K, Goh J, Chionh HL, Yap P: Coping strategies influence caregiver outcomes among Asian family caregivers of persons with dementia in Singapore. Alzheimer Dis Assoc Disord 2011; 25:34-41.

- 37 Stewart DE, Yuen T: A systematic review of resilience in the physically ill. Psychosomatics 2011;52: 199-209.

-38 Huang CY, Hsu MC, Chen TJ: An exploratory study of religious involvement as a moderator between anxiety, depressive symptoms and quality of life outcomes of older adults. J Clin Nurs 2012;21:609-619.

- 39 Cohen M, Werner P, Azaiza F: Emotional reactions of Arab lay persons to a person with Alzheimer's disease. Aging Ment Health 2009;13:31-37.

-40 Conry MC, Morgan K, Curry P, McGee H, Harrington J, Ward M, Shelley E: The clustering of health behaviours in Ireland and their relationship with mental health, self-rated health and quality of life. BMC Public Health 2011;11:692.

-41 Kontos EZ, Emmons KM, Puleo E, Viswanath K: Determinants and beliefs of health information mavens among a lower-socioeconomic position and minority population. Soc Sci Med 2011;73:22-32.

42 Department of Statistics of Singapore: Key Household Characteristics and Household Income Trends, 2011. http://www.singstat.gov.sg/pubn/papers/people.html\#householdincome (last accessed March 20, 2012).

-43 Sperling RA, Aisen PS: Toward defining the preclinical stages of Alzheimer's disease: recommendations from the National Institute on Aging-Alzheimer's Association workgroups on diagnostic guidelines for Alzheimer's disease. Alzheimers Dement 2011;7:280-292. 